\title{
Changes in Spike Protein Antibody Titer Over 90 Days after the Second Dose of SARS- CoV-2 Vaccine in Japanese Dialysis Patients
}

Haruki Wakai ( $\square$ harukiwakai@reiseikai.org )

Reiseikai Medical Corporation Shinagawa Garden Clinic

Natsumi Abe

Reiseikai Medical Corporation Shinagawa Garden Clinic

Touno Tokuda

Reiseikai Medical Corporation Shinagawa Garden Clinic

Rika Yamanaka

Reiseikai Medical Corporation Shinagawa Garden Clinic

\section{Satoshi Ebihara}

Reiseikai Medical Corporation Shinagawa Garden Clinic

\section{Kensuke Izumaru}

Reiseikai Medical Corporation Gotanda Garden Clinic

Daisuke Ishii

Kitasato University School of Medicine

\section{Toru Hyodo}

Kitasato University School of Medicine

Kazunari Yoshida

Kitasato University School of Medicine

\section{Research Article}

Keywords: SARS-CoV-2, COVID-19, vaccine, dialysis, antibody titer

Posted Date: December 14th, 2021

DOI: https://doi.org/10.21203/rs.3.rs-1165368/v1

License: (c) (i) This work is licensed under a Creative Commons Attribution 4.0 International License.

Read Full License 


\section{Abstract}

Objectives There is no report on antibody titers after vaccination against SARS-CoV-2 in Japanese dialysis patients. As dialysis is different between Japan and other countries, changes in antibody titers were examined.

Methods Baseline characteristics and anti-spike protein antibody titers (Roche) over 90 days after administration of the BNT162b2 messenger RNA vaccine were investigated in dialysis patients.

Results The maximum anti-spike protein antibody titer after the second dose was 737.9 (326.8 to 1143.4) and was reached at 19 (17 to 24.3) days after the second dose. Antibody titers decreased over time, with titers of 770 (316.4 to 1089) at 15 days, 385 (203 to 689.7) at 30 days, 253.5 (138 to 423) at 60 days, and 208 (107 to 375 ) at 90 days after the second dose. When an antibody titer of $137.052 \mathrm{U} / \mathrm{mL}$ was assumed to be a measure related to breakthrough infection, the proportion of subjects with antibody titers exceeding this level was $90.1 \otimes$ at 15 days, $85.3 \%$ at 30 days, $75.0 \%$ at 60 days, and $65.4 \%$ at 90 days after the second dose. When a decrease in antibody titers below the assumed breakthrough level was defined as an event, subjects with a pre-dialysis albumin $\geq 3.5$ were significantly less likely to experience an event than subjects with a pre-dialysis albumin $<3.5$. In addition, this study suggests that the presence of anti-spike protein levels $\geq 313$ at 30 days after the second vaccine dose might be a factor in maintaining antibody titers exceeding the assumed breakthrough level at 90 days after the second dose.

Conclusions The maximum antibody titers and the antibody titers at 90 days after the second vaccine dose in Japanese dialysis patients were approximately $30 \%$ of those in non-dialysis individuals reported otherwise, it was similar to those in dialysis patients in other countries. Whether an additional vaccine dose is needed should be determined based on indicators serving as factors in maintaining antibody titers as well as the status of the spread of infection.

\section{Introduction}

As of October 23, 2021, the coronavirus disease 2019 (COVID-19) pandemic caused by severe acute respiratory syndrome coronavirus 2 (SARS-CoV-2) has expanded to 216 countries in the world, with as many as approximately 242 million cases of infection and 4.9 million deaths occurring as of October 24 , $2021[1]$.

Dialysis patients have a higher risk of infections than non-dialysis patients. In the U.S. early in the 2000s, it was reported that dialysis patients have a 100 to 300 times higher sepsis-related mortality rate [2] and an approximately 15 times higher mortality rate related to pulmonary infection [3] than the general population. Then, the unadjusted mortality rate and adjusted mortality rate decreased by $26 \%$ and $28 \%$, respectively, in the dialysis population between 2001 and 2017 [4]. However, the risk of infection in 
dialysis patients remains high, and a report using data for the U.S. in 2016 indicates that the frequency of hospitalizations per 1000 person-years in individuals aged 65 years or older was approximately three times higher among patients with CKD than those without CKD, with cardiovascular events and infections accounting for $23 \%$ and $21 \%$, respectively, of such hospitalizations [5]. Also, in Japan, it was reported that the mortality rate of infections among dialysis patients was approximately eight times higher than that in the general population [6]. In addition, a survey on Japanese dialysis patients in 2019 showed that, among 31,905 patients with a documented cause of death and sex, $21.5 \%$ of the documented causes of death were caused by infections, the second most common cause of death after heart failure (22.7\%) [7]. These reports highlight that infectious disease control among dialysis patients is an issue.

The environment surrounding dialysis patients in Japan has different aspects from that in other countries. It was reported that the rate of internal shunt creation in Japan is $91 \%$, which is higher than the rates of $69 \%$ in Europe and $68 \%$ in the U.S., and that CRP levels are higher in other countries than those in Japan [8]. In addition, in Japan, catheters or artificial blood vessels, which carry a high risk for infection, are less likely to be used than in other countries [9]. Also, dialyzers are not reused, and the usage of intravenous irons is also lower than in other countries [10]. Robinson et al. reports that the risk of death among dialysis patients in other countries compared with Japan is 2.4 -fold and 2.8-fold, respectively, in terms of one-year survival and five-year survival rates [11].

Vaccines against SARS-CoV-2 have been developed by various pharmaceutical companies, with the vaccines manufactured by Pfizer, Moderna, AstraZeneca, and Janssen having gained widespread use. Antibody titers induced by these vaccines have been already well-documented in the general population. Although there are several published reports on changes in vaccine antibody titers among dialysis patients outside of Japan, changes in vaccine antibody titers among dialysis patients in Japan may be different from those in other countries. Thus, we considered it significant to examine changes in antibody titers among Japanese dialysis patients.

As of October 23, 2021, among approximately 344,000 dialysis patients in Japan [7], only 2,644 patients, approximately $0.8 \%$, were infected [12]. A total of $412(16 \%)$ dialysis patients have died after infection and 1056 patients (39.9\%) had an unknown outcome [12]. We might say that strict control by the government, public, and medical institutions has led to favorable outcomes in both the prevention of the spread of infection and treatment after infection. Therefore, we add to data on antibody titers of vaccine to contribute to infection prevention.

\section{Materials And Methods}




\section{Ethic approval and consent to participate}

This study was approved by the ethics committee of Reiseikai Medical Corporation (July 2nd, 2021) and conducted in accordance with the Declaration of Helsinki and the ethical guidelines provided by the Ministry of Health, Labour and Welfare. As this study involved no invasion or intervention to patients and used only information such as medical information and residual samples, an opt-out was provided to disclose information, and an opportunity for refusal was assured. This study is registered with the Clinical Trials Registry (UMIN000046281).

\section{Study design}

This study was a collaborative observational study at two centers. Patients were included in this study when they met all of the following inclusion criteria and did not meet any of the following exclusion criteria (with the rationales presented in parentheses).

Inclusion criteria

1) Dialysis patients who regularly visited the study center (in order to track data).

2) Patients who received the first vaccine dose in June 2021 (to examine antibody titers induced by vaccination).

\section{Exclusion criteria}

1) Patients who have been infected with SARS-CoV-2 (to understand the actual situation in uninfected individuals because many Japanese people have not been infected).

2) Patients who received only one vaccine dose (because antibody titers do not increase without two vaccine doses).

\section{Method}

anti-spike protein (Anti-S) IgM antibody titers (assay reagent: SARS-CoV-2 IgM assay reagent (IB), assay system: “Lumipulse ${ }^{\circledR}$ L2400" and "Lumipulse Presto®," FUJIREBIO Inc., Tokyo, Japan), anti-S IgG antibody titers (assay reagent: SARS-CoV-2 S-IgG assay reagent (IB), assay system: "Lumipulse® L2400" and "Lumipulse Presto\&," FUJIREBIO Inc., Tokyo, Japan), and anti-spike protein antibody titers (Elecsys Anti-SARS-CoV-2 S antibody qualitative test reagent, Roche Diagnostics K.K., Tokyo, Japan) to SARS-CoV- 
2 were measured approximately once every two weeks after the first dose of the BNT162b2 messenger RNA vaccine (Pfizer-BioNTech) (hereinafter referred to as "BNT162b2 vaccine"). Antibody levels were measured using residual samples of the blood collected for routine tests at dialysis sessions. The reagents used for the measurement of antibody titers and the timing of measurement are shown in Figure 1. Although Fujirebio's reagents were originally used for examining changes in both anti-S IgM antibody titers and anti-S IgG antibody titers, they were then replaced with Roche's assay of anti-spike protein antibody titers for the following two reasons. One reason is that, although some samples showed undetectable anti-S IgG antibody titers as determined by Fujirebio's assay, such measured values were required to be evaluated as specific values that were not below the limit of detection. The other reason is that the use of measured values obtained by Roche's assay, which is widely used worldwide, provided comparability with other study results. Major results obtained from this study were expressed as antispike protein antibody titers obtained using Roche's reagent.

\section{Survey items}

Baseline subject demographics included age, sex, underlying disease(s), status of comorbid diabetes, height, body weight (post-dialysis), Body Mass Index (BMI), history of dialysis, creatinine index, C-reactive protein assay, HbA1c (NGSP), PTH-intact, glycated albumin, ferritin, geriatric nutritional risk index, Kt/V (shinzato), protein catabolism rate, Transferrin saturation, albumin (pre- and post-dialysis) and clear space ratio. (Unless otherwise specified, the last pre-dialysis values before the first vaccine dose are shown.)

Anti-S IgM antibody titers, anti-S IgG antibody titers, and anti-spike protein antibody titers after vaccination were tested approximately once every two weeks after the first vaccine dose, coinciding with blood collection for routine tests, and values up to approximately 90 days were evaluated (Figure 1).

As this study was designed to examine antibody titers over a long period of time, these endpoints concern only this report, and the study continues further.

\section{Endpoints}

The endpoints for this report are shown below:

1. Antibody titers at 90 days ( \pm 10 days) after the second dose (the primary endpoint) 
2. Maximum anti-spike protein antibody titers after the second dose and the number of days until the day with the maximum antibody titer

3. Antibody titers at 15 days ( \pm 4 days), 30 days ( \pm 10 days), and 60 days ( \pm 10 days) after the second dose

Antibody titers at 30 days were evaluated in the cases of both a) converted values excluded and b) values converted from anti-S IgG antibody titers being imputed in the absence of available anti-spike protein antibody titers.

4. Univariate and multivariate analyses of factors affecting maximum anti-spike protein antibody titers after the second dose

5. Univariate and multivariate analyses of factors affecting anti-spike protein antibody titers at 90 days after the second dose

6. Univariate and multivariate analyses of factors contributing to anti-spike protein antibody titers > $137.052 \mathrm{U} / \mathrm{mL}$ at 90 days after the second dose, and exploratory analysis based on these results

هChloe's report indicated that the protection was $89.3 \%$ with antibody titers $>141 \mathrm{BAU}$ and $12.4 \%$ with antibody titers of 13 to $141 \mathrm{BAU}$ [13]. This antibody titer of $141 \mathrm{BAU}$ corresponding to $89.3 \%$ protection was converted to $141 * 0.972 \mathrm{BAU}=137.052 \mathrm{U} / \mathrm{mL}$ using the conversion formula based on the report by Krzysztof et al. [14], which was assumed as a measure related to breakthrough infection. An analysis was performed based on whether this level was exceeded. An antibody titer of $137.052 \mathrm{U} / \mathrm{mL}$ is hereinafter

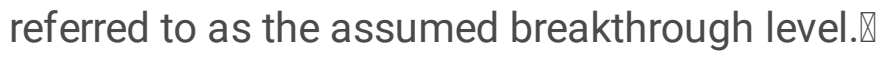

7. Predictive performance of anti-spike protein antibody titers at 30 days for anti-spike protein antibody titers at 90 days exceeding the assumed breakthrough level

8. Correlation between anti-S IgM antibody titers and anti-S IgG antibody titers (for maximum values [i] at each time point and [ii] for each subject)

9. Maximum anti-S IgM antibody titers and the number of days until the day with the maximum antibody

10. Correlation between anti-S IgG antibody titers by Fujirebio's assay and anti-spike protein antibody titers by Roche's assay

It should be noted that in the absence of available anti-spike protein antibody titers, anti-spike protein antibody titers were calculated from anti-S IgG antibody titers using the approximation formula obtained after the test of correlation as listed in 10) and imputed for evaluation. The conversion formula was shown in 10). 


\section{Statistical analyses}

Data analysis was performed using R version 3.5.2 (R Foundation for Statistical Computing, Vienna, Austria [https://www.R-project.org/]) and for Excel version 3.21 (Social Survey Research Information Co., Ltd., Tokyo, Japan). In the analyses of factors affecting anti-spike protein antibody titers (maximum values and values at 90 days), correlations between anti-spike protein antibody titers and various measured values were tested using Spearman's test, and differences in anti-spike protein antibody titers between two groups defined by nominal variables were tested using the Wilcoxon rank sum test. In addition, in the multiple regression analyses including anti-spike protein antibody titers as an object variable, the normality of residual errors in the multiple regression analyses was tested using the ShapiroWilk test to determine its appropriateness. In the analyses of factors contributing to anti-spike protein antibody titers $>137.052 \mathrm{U} / \mathrm{mL}$ at 90 days, the Student's t-test and Chi-squared test were used for continuous variables and nominal variables, respectively, in univariate analysis, while the Wald Chisquared test was used in multiple logistic regression analysis. Correlations between anti-S IgM antibody titers and anti-S IgG antibody titers and between anti-S IgG antibody titers and anti-spike protein antibody titers were tested using Spearman's test. As the latter was also used for conversion using an approximation formula, Pearson's test correlation coefficient was also verified. In preparing a KaplanMeier curve, comparisons were made using the log-rank test and generalized Wilcoxon test. A two-sided significance level of $5 \%$ was used, and the analytical results in text were expressed as mean \pm standard deviation or median ( $1^{\text {st }}$ quartile to $3^{\text {rd }}$ quartile).

\section{Results}

Baseline subject demographics are shown in Table 1. A total of 96 patients met the eligibility criteria. They had an age of $68.5 \pm 15.2$ years (70.5 [ 57 to 81.25] years), consisted of 72 men (75\%) and 24 women $(25 \%)$, and had a duration of dialysis of $5.4 \pm 5.3$ years.

\section{Endpoints 1) to 3)}

Figure 2 shows the maximum anti-spike protein antibody titers after the second vaccine dose, and antispike protein antibody titers at $15,30,60$, and 90 days after the second vaccine dose. The maximum antispike protein antibody titer after the second dose was 737.9 (326.8 to 1143.4) and was reached at 19 (17 to 24.3) days after the second dose, with two patients showing a persistent increase in antibody titers until the last day of measurement, including one patient who showed no significant increase in anti-spike protein antibody titers (Supp. 1). Anti-spike protein antibody titers decreased over time, with titers of 770 (316.4 to 1089) at 15 days, 385 (203 to 689.7) at 30 days, 253.5 (138 to 423) at 60 days, and 208 (107 to $375)$ at 90 days after the second dose. The proportion of patients showing titers exceeding the assumed breakthrough level was $90.1 \%$ at 15 days, $85.3 \%$ at 30 days, $75.0 \%$ at 60 days, and $65.4 \%$ at 90 days after the second dose. Changes in anti-S IgG antibody titers are shown in Supp. 2-a and b. In the majority of 
subjects, anti-S IgG antibody titers were undetectable after the first dose and before the second dose, generally peaked at three weeks after the second dose, and then declined. In addition, three patients still showed undetectable anti-S IgG antibody titers after the second dose, but had measurable, although very low, anti-spike protein antibody titers when tested using the same samples.

\section{Endpoint 4)}

The results of the analyses of factors affecting maximum anti-spike protein antibody titers are shown in Supp. 3. The univariate analysis showed that high pre-vaccination creatinine index, high pre-vaccination pre-dialysis albumin, and an age younger than 70 years were the significant factors associated with high antibody titers (Supp. 3-a and b). A multiple regression analysis with the factors identified as significant by the univariate analysis, age, $\mathrm{BMI}$, and history of dialysis as the explanatory variables was performed, but with no normal distribution of residual errors obtained (Supp. 3-c). Thus, this multiple regression analysis was not appropriate, and another multiple regression analysis with log anti-spike protein antibody titers as the object variable was therefore performed, but again with no normal distribution of residual errors obtained (Supp. 3-d).

\section{Endpoint 5)}

The results of the analyses of factors affecting anti-spike protein antibody titers at 90 days after the second vaccine dose are shown in Supp. 4. The univariate analysis identified no significant factors associated with high anti-spike protein antibody titers. The factors with a $p$ value $<0.1$ were BMI $(\rho=-0.194, p=0.082), K t / V(\rho=0.213, p=0.056)$, and clear space ratio $(\rho=0.211, p=0.059)$ (Supp. 4-a). The multiple regression analysis with these three factors as well as age, sex, and history of dialysis as the explanatory variables was performed, but with no normal distribution of residual errors obtained (Supp. 4c). Thus, this multiple regression analysis was not appropriate, and another multiple regression analysis with log anti-spike protein antibody titers as the object variable was therefore performed, but again with no normal distribution of residual errors obtained (data not shown).

\section{Endpoint 6)}

The univariate analysis of factors associated with anti-spike protein antibody titers at 90 days after the second vaccine dose exceeding the assumed breakthrough level showed that high pre-dialysis albumin and a young age were the significant factors (Table 2-a and b). The multiple logistic regression analysis with these two factors as well as sex, BMI, and history of dialysis as the explanatory variables showed that high pre-dialysis albumin and a young age were the significant factors, consistent with the univariate analysis (Table 3). In addition, in light of these results, a Kaplan-Meier curve using an anti-spike protein 
antibody titer below $137.052 \mathrm{U} / \mathrm{mL}$ as an event in the two groups defined by pre-dialysis albumins of $\geq$ $3.5 /<3.5$ was created and showed that the group with pre-dialysis albumins $\geq 3.5$ was less likely to experience an event (Figure 3).

\section{Endpoint 7)}

The predictive performance of anti-spike protein antibody titers at 30 days after the second vaccine dose for anti-spike protein antibody titers at 90 days after the second vaccine dose exceeding the assumed breakthrough level was analyzed using a receiver operating characteristic (ROC) curve. Results showed that the cut-off value was $313.0 \mathrm{U} / \mathrm{mL}$, and the area under the curve (AUC) was 0.925 (Figure 4). When values converted from anti-S IgG antibody titers were included, the cut-off value was $312.0 \mathrm{U} / \mathrm{mL}$, and the AUC was 0.921.

\section{Endpoint 8)}

The correlation between anti-S IgM antibody titers and anti-S IgG antibody titers is shown in Supp. 5. The correlation coefficient at each time point was $p=0674$. ( $p<0.001$, Supp5-a), and a significant positive correlation was observed with a correlation coefficient for the maximum value of $\rho=0.458(p=0.004$, Supp5-b) for each patient.

\section{Endpoint 9)}

The maximum anti-S IgM antibody titer was 1 ( 0.5 to 2.3 ) and was reached at 17 (7 to 19$)$ days after the second vaccine dose (Supp. 6).

\section{Endpoint 10)}

Evaluation of anti-S IgG antibody titers and anti-spike protein antibody titers obtained from the same 146 samples showed a strong positive correlation between two types of antibody titers $(\rho=0.864, p<0.001$, Spearman's test. $r=0.900, p<0.001$, Pearson's test. Supp. 7). Data with only anti-S IgG antibody titers available due to failure to test anti-spike protein antibody titers were imputated with values calculated using the approximation formula "anti-spike protein antibody titer $(\mathrm{U} / \mathrm{mL})=9.7246 \times$ anti-S IgG antibody titer $(\mathrm{AU} / \mathrm{mL})$ - 19.614." (Measured anti-S IgG antibody titers $<2.02$ were observed in three patients at 15 days and two patients at 30 days and were all $<1$. For Endpoint 3 , these measured values were handled as 0 .) 


\section{Discussion}

\section{Comparison with reports on Japanese individuals without dialysis ( 30 and 90 days after the second vaccine dose)}

This study showed that the anti-spike protein antibody titers among Japanese dialysis patients were 385 (203 to 689.7 ) $\mathrm{U} / \mathrm{mL}$ at 30 days and 208 (107 to 375 ) $\mathrm{U} / \mathrm{mL}$ at 90 days after the second vaccine dose, which were lower at both time points than the reports based on data from Japanese individuals without dialysis. A study of 2015 subjects previously reported that the median anti-spike protein antibody titer at 30 days was 2,060 U/mL [15], which represented an approximately 3-fold difference from the median maximum titer of $737.9 \mathrm{U} / \mathrm{mL}$ observed in individual subjects up to 30 days after the second vaccine dose in this study. In addition, the antibody titers at 90 days after the second vaccine dose have been reported to be 764 (423 to 1140) $\mathrm{U} / \mathrm{mL}$ in a study of 378 subjects [16], which also represented an approximately 3 -fold difference from the result from this study. This study suggested that anti-spike protein antibody titers among dialysis patients were lower than those among individuals without dialysis both at 30 days after the second vaccine dose and during the subsequent phase of decline. Hemodialysis patients were previously reported to have a reduced immune response [17], supported by our results. It is also reported that Japanese individuals without dialysis show a decrease in the median antibody titer to 28.6\% between two weeks and three months after the second dose when using FUJIFILM's test reagent [18]. Although the schedule was not strictly managed due to the observational nature of this study, a comparison with the value at $15 \pm 4$ days shows a decrease to $26.9 \%$, suggesting that a percent decrease is almost the same with and without dialysis.

\section{Comparison with overseas reports on dialysis patients (around 30 days after the second dose)}

A Germany study report using the same anti-spike protein antibody titer assay reagents as used in this study showed that anti-spike protein antibody titers were significantly lower in subjects with dialysis $(253.5 \mathrm{U} / \mathrm{mL})$ than in subjects without dialysis $(1756 \mathrm{U} / \mathrm{mL})$ [19]. These values in the Germany report are slightly lower than the antibody titers in this study. This is likely attributed to older age in the Germany study [75 (64 to 82 ) years] compared to this study [70.5 (57 to 81.25) years].

A French study of 69 subjects using the same reagent showed that the anti-spike protein antibody titers after the second dose were 284 (83 to 1190) U/mL [20]. Although these values are slightly lower than those in this study, no specific subject demographic variable explaining this difference was identified. A difference in the environment surrounding dialysis could contribute to this difference. 
A study using Abbott's reagent [21] reported the antibody titer of $276 \mathrm{AU} / \mathrm{mL}$ (IQR: 83.4 to 526.6 ) in dialysis patients compared with the antibody titer of $1082 \mathrm{AU} / \mathrm{mL}$ (735.0 to 1662.0) in individuals without dialysis, representing an approximately 3-fold difference, as observed in our study. Since the result of this study is consistent with our data, it may support our conclusion.

A Germany study comparing dialysis patients and individuals without dialysis using DiaSorin's reagent (for anti-S IgG antibody titers) reported that individuals younger than 60 years old, even with dialysis, showed the same level of antibody titers as that in individuals without dialysis [22]. In their report, differences are also likely to have arisen due to the upper limit of detection of the test reagent. It is likely that different reagents result in slightly different analytical results.

\section{Factors predisposed to an increase in antibody titers}

The univariate analysis showed that high creatinine index, high pre-dialysis albumin, and an age younger than 70 were the factors associated with high maximum anti-spike protein antibody titers. The multiple regression analyses were performed, but with the normality of residual errors rejected. Therefore, the model was considered inappropriate, and discussions were made based on the results of the univariate analysis.

Previous studies on dialysis patients have also previously reported that high albumin is a factor associated with high antibody titers [20,21, 23], which was also confirmed in Japanese dialysis patients by this study.

Younger age has also been previously reported to be associated with higher antibody titers [20, 21, 24]. This study analyzed the subgroups defined by age ( $\geq 60 /<60$ years, $\geq 65 /<65$ years, $\geq 70 /<70$ years, and $\geq 75 /<75$ years) and found a significant difference in the comparison of ages $\geq 70$ years and $<70$ years, but the threshold in determining whether there is a significant difference may affect the population. However, as tests of correlation also showed a tendency toward negative correlations, it is considered sure that antibody titers are less likely to increase among older individuals.

There is no previous report on creatinine index and anti-spike protein antibody titers. Creatinine index reflects muscle mass. As muscle mass decreases, the body fluid compartment decreases, resulting in violent fluctuations in circulating blood volume associated with changes in total body water. In recent years, a modified creatinine index [25], a simplified version of the creatinine index, which is calculated by 
only age, sex, pre-dialysis serum creatinine level, and Kt/ $\mathrm{V}$ for urea, has been proposed, and Arase et al. reported that a low modified creatinine index was associated with increased long-term risk of infectionrelated mortality in dialysis patients [26]. A larger skeletal muscle mass is associated with a higher creatinine index, and skeletal muscles have multilateral functions on immunity. Skeletal muscles are the most important organ that induces heat production [27] and play a key role in energy storage [28]. When acquiring an infection, individuals experience frequent muscle contractions, mainly during tremors, to increase body temperature for enhancing immunity [29]. Thermotherapy reduces viral and bacterial growth while promoting the migration and function of leukocytes and macrophages. A decrease in skeletal muscle brings about inadequate heat production and energy consumption, potentially causing an increase in the severity of infections.

Other reported factors leading to a predisposition to an increase in antibody titers among dialysis patients include previous infection [19, 24], duration of dialysis of < 5 years [23], high Kt/V [21], absence of the use of immunosuppressants, high white blood cell counts, and high gamma globulin levels [20]. Although these factors did not affect maximum anti-spike protein antibody titers in this study, different results may be obtained depending on the characteristics of the population.

Reported factors associated with high antibody titers among individuals undergoing dialysis include a young age $[15,18,30]$, female sex $[15,18]$, previous infection with SARS-CoV-2 [15, 31], absence of comorbid diabetes [32], intervals of 18 to 25 days between the first and second doses, current intake of anti-allergic agents, absence of the intake of immunosuppressants/steroids, and absence of a drinking habit [15]. Regarding gender, given that males accounted for $75 \%$ of the subject population in this study, it is difficult to make an accurate comparison unless the ratio of females is increased. Other factors will be reviewed in further reports published in various countries or regions.

\section{Factors in maintaining antibody titers that are not expected to cause breakthrough infection}

Reports on individuals other than dialysis patients describe factors that maintain high antibody titers that include a young age [16,33], the absence of smoking [16, 33], female sex [33], and previous infection [34]. This study failed to identify significant factors associated with high anti-spike protein antibody titers at 90 days. Due to large variability in antibody titers, it is considered difficult to make evaluations using absolute values. In contrast, higher antibody titers than necessary are not required if antibody titers associated with breakthrough infection are maintained. Thus, the value corresponding to $89.3 \%$ protection as reported by Chloe [13] was converted to the antibody titer in this study [14], and an antibody titer of $137.052 \mathrm{U} / \mathrm{mL}$ (assumed breakthrough level) was assumed as a measure related to breakthrough infection. Factors associated with antibody titers exceeding this level at 90 days were analyzed. Both 
univariate and multivariate analyses showed that high albumin and a young age were identified as the factors associated with anti-spike protein antibody titers exceeding the assumed breakthrough level. Although the mechanism underlying the effects of these factors is the same as discussed for the factors associated with increased maximum antibody titers, the Kaplan-Meier curve prepared as an explanatory analysis comparing albumin levels $\geq$ and $<3.5$ suggested that the presence of albumin levels $\geq 3.5$ might be an indicator of antibody titers kept above the assumed breakthrough level.

For the BNT162b2 vaccine, a study evaluating anti-SAb IgG antibody titers using Siemens' reagent in dialysis patients reported that $8.1 \%(19 / 235)$ of subjects with an antibody titer $\geq 20 \mathrm{U} / \mathrm{L}$ (the upper limit of assay of Siemens' reagent) at two months after vaccination had undetectable antibody titers by 180 days, whereas $61.1 \%(80 / 131)$ of subjects with the first measured antibody titer $<20 \mathrm{U} / \mathrm{L}$ had undetectable antibody titers by 180 days [35], suggesting that anti-spike protein antibody titers at 90 days may exceed the assumed breakthrough level when anti-spike protein antibody titers at 30 days were not less than $313 \mathrm{U} / \mathrm{mL}$. Although repeated measurements of vaccine antibody titers cause the burdens of expense and blood collection, such burdens will be reduced if a measurement at 30 days estimates subsequent maintenance.

Although this study used Chloe's report, there is no definitive report on antibody titers to prevent breakthrough infection. There are reports indicating that breakthrough infection occurred despite high neutralizing titers being maintained [36, 37]. The use of different test reagents between the studies makes it difficult to make comparisons, and potential mutations of the virus make it impossible to determine what level of antibody titers is adequate. However, currently available data may guide decision making.

In a six-month study in 1567 dialysis patients using Siemens' reagent, anti-S IgG antibodies to the Ad26.COV2. S vaccine (Janssen Pharmaceutical K.K.) remained low from the beginning, median antibody titers to the BNT162b2 vaccine were decreased to approximately $1 / 6$ at six months, and antibody titers to mRNA-1273 (Moderna Inc.) remained higher [35]. As our study included only individuals who received the BNT162b2 vaccine, caution should be exercised because the persistence of antibody titers varies with the type of vaccine. As our study also has only the ability to predict antibody titers up to 90 days after the second vaccine dose, further study is warranted to examine more long-term maintenance of antibody titers.

In addition, all is not lost in the event of a breakthrough infection. There is a previous report that patients with breakthrough infections all had mild cases of the disease [36]. Although, as a matter of course, some reports indicated that cases other than mild ones were observed [37], memory T-cells that persist in the 
body may enhance immune response associated with infection. Although this is only hypothetical because of the lack of currently available methods of measurement of memory T-cells, everything is not determined exclusively by vaccine antibody titers.

\section{Anti-S IgM antibody titers and anti-S IgG antibody titers}

Anti-S IgM antibody titers reached a peak slightly earlier than anti-S IgG antibody titers. In addition, there was a significant positive correlation between both types of antibody titers at each time point. Moreover, tests of correlations were performed using maximum values from individual subjects in consideration of a gap in timing with maximum values, and again demonstrated a significant positive correlation. In contrast, three subjects with undetectable anti-S IgG antibody titers also showed detectable anti-S IgM antibody levels (ranging from 0.1 to 0.2 ). Although there is a correlation between anti-S IgM antibody titers and anti-S IgG antibody titers, this study suggested that some subjects showed no IgG response.

\section{Subjects with no increase in IgG antibody titers}

As described above, three subjects had no increase in anti-S IgG antibody titers. A French study on dialysis patients previously reported that three of 69 patients showed undetectable anti-spike protein antibody titers after the second vaccine dose [20]. However, their report indicated that such undetectable antibody titers were attributable to previous immunological diseases. In addition, one of the three patients had an increase in antibody titers with the third vaccine dose. There are other reports that also indicated that patients who received a transplant using immunosuppressants did not develop antibodies [38]. In contrast, there is also a report that some patients showed no increase in antibody titers even in the absence of immunological diseases or use of immunosuppressants [39]. In our study, the T-cell response test ("T-SPOT $®$ Discovery SARS-CoV-2", RIKEN GENESIS CO., LTD., Tokyo, Japan) was performed on three subjects who never had an increase in anti-S IgG antibody titers, but two of the three subjects had no response and the remaining subject had almost no response. It should be noted that some subjects were unresponsive and inactivated. In addition, a comparison with the other subjects identified no specific laboratory parameters or patient demographics. These three patients will be followed up for a longer period of time and closely monitored for responses if they receive the third vaccine dose.

\section{Subjects with a persistent increase in antibody titers}

Two subjects had the highest antibody titers at 90 days after the second vaccine dose. One had an undetectable anti-S IgG antibody level of $2.45 \mathrm{U} / \mathrm{mL}$, and the other had a consistent increase in antibody titers, starting from 74 to $103,224,347,414$, and $493 \mathrm{U} / \mathrm{mL}$. There was no difference between the 
subjects in terms of subject demographics. Although no cause of persistent increase in antibody titers was identified, it should be noted that some subjects reached a peak later.

\section{Limitation}

This study has several limitations.

One of them is that $\mathrm{N}$ antibodies were measured in all samples before vaccination. Thus, in consideration of potential subclinical infection, nine subjects with anti-S IgG antibody titers not below the limit of detection before the second dose were tested for $\mathrm{N}$ antibodies approximately four months after the first vaccine dose, and all nine were found to be negative. However, the possibility cannot be ruled out that $\mathrm{N}$ antibodies became undetectable after vaccination. Thus, an additional analysis excluding these nine subjects was performed, but the results were not significantly different. Although the Roche's test kit was not used in the initial stage of this study, the presence of converted values is another limitation. In this respect, both analyses including and not including converted values were performed for examination and demonstrated similar results (data not shown). In addition, as this study was an observational study using residual samples, tests were not strictly scheduled. Thus, for analysis, an appropriate period was set, and data closest to the reference date in the period were included, but data from some patients were outside the appropriate period, resulting in missing data. In addition, this study used the value corresponding to $89.3 \%$ protection based on Chloe's report as the assumed breakthrough level, but this assumed breakthrough level is not an absolute value but just a reference value. It should be noted that this level is positioned as only a current guide of SARS-CoV-2, which will undergo further mutation. As this study was performed at two centers in Tokyo, Japan, our results cannot be uniformly applied to different environments, such as regions and equipment used at centers.

\section{Conclusions}

The maximum antibody titers and the antibody titers at 90 days after the second vaccine dose in Japanese dialysis patients were approximately $30 \%$ of those in non-dialysis individuals reported otherwise, without significant differences with those in dialysis patients in other countries. As dialysis patients have lower antibody titers, it may be necessary for them to receive the third vaccine dose early. Whether an additional vaccine dose is needed should be determined based on antibody titers at 30 days, factors that maintain antibody titers, as well as indicators such as albumin and age, and the status of infection spread/reduction.

\section{Declarations}

\section{Availability of data and material}


The biochemical data used to support the findings of this study are available from the corresponding author upon request.

\section{Acknowledgments}

We would like to thank the physicians and staff of Reiseikai Medical Corporation Shinagawa Garden Clinic and Gotanda Garden Clinic in this study.

\section{Funding}

This study was self-funded.

\section{Author information}

Affiliations

Reiseikai Medical Corporation Shinagawa Garden Clinic, Imasu Ohsaki Building 2F, 1-20-3 Ohsaki, Shinagawa, Tokyo 141-0032, Japan.

Haruki Wakai, Natsumi Abe, Touno Tokuda, Rika Yamanaka, Satoshi Ebihara

Reiseikai Medical Corporation Gotanda Garden Clinic, Kanpai Building 7F, 5-22-27 Higashi-Gotanda, Shinagawa, Tokyo 141-0022, Japan

Kensuke Izumaru

Kitasato University School of Medicine, 1-15-1 Kitasato, Minami-ku, Sagamihara 252-0374, Kanagawa, Japan.

Daisuke Ishii, Toru Hyodo, Kazunari Yoshida

\section{Contributions}


Conceived and designed the experiments: HW, KI, DI, TH, KY. Enrolled patients: HW, NA, TT, RY, SE, KI, DI, TH, KY. Analyzed the data: HW. Wrote the first draft of the manuscript: HW. Contributed to the writing of the manuscript: HW, KI, DI, TH, KY. All authors agreed with the manuscript's results and conclusions. All authors have read, and confirm that they meet, ICMJE criteria for authorship.

\section{Corresponding author}

Correspondence to Haruki Wakai.

\section{Ethics declarations}

\section{Ethics approval and consent to participate}

This study was approved by the ethics committee of Reiseikai Medical Corporation (July 2nd, 2021) and conducted in accordance with the Declaration of Helsinki and the ethical guidelines provided by the Ministry of Health, Labour and Welfare. As this study involved no invasion or intervention to patients and used only information such as medical information and residual samples, an opt-out was provided to disclose information, and an opportunity for refusal was assured. This study is registered with the Clinical Trials Registry (UMIN000046281).

\section{Consent for publication}

Not applicable.

\section{Competing Interests}

The authors declare no conflicts of interest.

\section{Abbreviations}

COVID-19: coronavirus disease 2019; SARS-CoV-2: severe acute respiratory syndrome coronavirus 2; AntiS: anti-spike protein; BMI: Body Mass Index; ROC: receiver operating characteristic; AUC: area under the curve.

\section{References}


1. World Health Organization, Coronavirus disease (COVID-19) pandemic. WHO. Available at: https://www.who.int/emergencies/diseases/novel-coronavirus-2019. Accessed on 24/10/2021.

2. Sarnak MJ, Jaber BL. Mortality caused by sepsis in patients with end-stage renal disease compared with the general population. Kidney Int. 2000;58:1758-64,

3. Sarnak MJ, Jaber B. Pulmonary infectious mortality among patients with end-stage renal disease. Chest. 2001;120:1883-87

[4] US Renal Data System 2019 Annual Data Report: Epidemiology of Kidney Disease in the United States. National Institute of Diabetes and Digestive and Kidney Diseases.

https://www.usrds.org/media/2371/2019-executive-summary.pdf Accessed on 24/10/2021.

[5] Saran R, Robinson B, Abbott KC, Agodoa LY, Ayanian J, Bragg-Gresham J, et al. Epidemiology of kidney disease in the United Statees. Am J Kidney Dis. 2017;69(3S1):A7-A8.

[6] Wakasugi M, Kawamura K, Yamamoto S, Kazama JJ, Narita I. High mortality rate of infectious diseases in dialysis patients $₫ A$ comparison with the general population in Japan. Therapeutic Apheresis and Dialysis 2012;16:226-31.

[7] Nitta K, Masakane I, Hanafusa N, Hoshino J, Taniguchi M, Joki N, et al. 2019 Annual Dialysis Data Report, JSDT Renal Data Registry Jpn Soc Dial Ther. 2020;53:579-632

8. Robinson BM, Akizawa T, Jager KJ, Kerr PG, Saran R, Pisoni RL. Factors affecting outcomes in patients reaching end-stage kidney disease worldwide: differences in access to renal replacement therapy, modality use, and haemodialysis practices. Lancet. 2016;388(10041):294-306.

[9] Ethier J, Mendelssohn DC, Elder SJ, Hasegawa T, Akizawa T, Akiba T, et al. Vascular access use and outcomes\an international perspective from the Dialysis Outcomes and Practice Patterns Study. Nephrol 
Dial Transplant. 2008;23:3219-3226.

[10] Pisoni RL, Bragg-Gresham JL, Young EW, Akizawa T, Asano Y, Locatelli F, et al. Anemia management

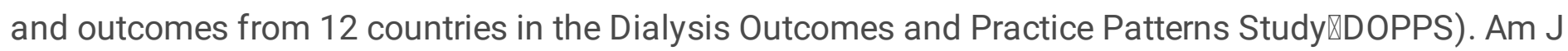
Kidney Dis. 2004;44:94-111.

[11] Robinson BM, Bieber B, Pisoni RL, Port FK. Dialysis Outcomes and Practice Patterns Study (DOPPS): its strengths, limitations, and role in informing practices and policies. Clin J Am Soc Nephrol. 2012;7:1897-905.

[12] Japanese Society for Dialysis Therapy. Available at: https://www.jsdt.or.jp/info/3406.html Accessed on $24 / 10 / 2021$.

[13] Dimeglio C, Herin F, Martin-Blondel G, Miedougé M, Izopet J. Antibody titers and protection against a SARS-CoV-2 infection. J Infect. 2021;S0163-4453(21)00483-7.

[14] Lukaszuk K, Kiewisz J, Rozanska K, Podolak A, Jakiel G, Woclawek-Potocka I, et al. Is WHO International Standard for Anti-SARS-CoV-2 Immunoglobulin Clinically Useful? doi: https://doi.org/10.1101/2021.04.29.21256246

[15] Kageyama T, Ikeda K, Tanaka S, Taniguchi T, Igari H, Onouchi $Y$, et al. Antibody responses to BNT162b2 mRNA COVID-19 vaccine in 2,015 healthcare workers in a single tertiary referral hospital in Japan

Available at: https://www.medrxiv.org/content/10.1101/2021.06.01.21258188v1 Accessed on 2/5/2021

[16] Nomura Y, Sawahata M, Nakamura Y, Kurihara M, Koike R, Katsube O, et al. Age and smoking predict antibody titres at 3 months after the second dose of the BNT162b2 COVID-19 vaccine. Available at: https://www.medrxiv.org/content/10.1101/2021.08.06.21261590v1 Accessed on 7/8/2021 
[17]Grupper A, Sharon N, Finn T, Cohen R, Israel M, Agbaria A, et al. Humoral response to the Pfizer BNT162b2 vaccine in patients undergoing maintenance hemodialysis. Clin J Am Soc Nephrol 2021;16:1037-42.

[18]Fujita Health University. Available at: https://www.fujita-hu.ac.jp/news/j93sdv000000b3zd.html. Accessed on 25/8/2021.

[19]Paal M, Arend FM, Lau T, Hasmann S, Soreth-Rieke D, Sorodoc-Otto J, et al. Antibody response to mRNA SARS-CoV-2 vaccines in haemodialysis patients. Clin Kidney J. 2021;14:2234-38

[20]Bensouna I, Caudwell V, Kubab S, Acquaviva S, Pardon A, Vittoz N, et al. SARS-CoV-2 Antibody Response After a Third Dose of the BNT162b2 Vaccine in Patients Receiving Maintenance Hemodialysis or Peritoneal Dialysis. Am J Kidney Dis. 2021:S0272-6386(21)00833-7.

[21]Danthu C, Hantz S, Dahlem A, Duval M, Ba B, Guibbert M, et al. Humoral Response after SARS-CoV-2 mRNA Vaccination in a Cohort of Hemodialysis Patients and Kidney Transplant Recipients. J Am Soc Nephrol. 2021;32:2153-58.

[22] Jahn M, Korth J, Dorsch O, Anastasiou OE, Sorge-Hädicke B, Tyczynski B, et al. Humoral Response to SARS-CoV-2-Vaccination with BNT162b2 (Pfizer-BioNTech) in Patients on Hemodialysis. Vaccines (Basel). 2021;9:360.

[23]Anand S, Montez-Rath ME, Han J, Garcia P, Cadden L, Hunsader P, et al. Antibody Response to COVID19 Vaccination in Patients Receiving Dialysis. J Am Soc Nephrol. 2021 ;32:2435-38.

[24]Tylicki L, Biedunkiewicz B, Dąbrowska M, Ślizień W, Tylicki P, Polewska K, et al. Humoral response to SARS-CoV-2 vaccination promises to improve the catastrophic prognosis of hemodialysis patients as a result of COVID-19: the COViNEPH Project. Pol Arch Intern Med. 2021 Sep 30;131(9):797-801. 
[25]Canaud B, Granger Vallée A, Molinari N, Chenine L, Leray-Moragues H, Rodriguez A, et al. Creatinine index as a surrogate of lean body mass derived from urea $\mathrm{Kt} / \mathrm{V}$, pre-dialysis serum levels and anthropometric characteristics of haemodialysis patients. PLoS One. $2014 ; 9:$ e93286.

26. Arase $\mathrm{H}$, Yamada S, Hiyamuta $\mathrm{H}$, Taniguchi M, Tokumoto M, Tsuruya K, et al. Modified creatinine index and risk for long-term infection-related mortality in hemodialysis patients: ten-year outcomes of the Q-Cohort Study. Sci Rep. 2020;10:1241.

27. Periasamy M, Herrera JL, Reis FCG. Skeletal muscle thermogenesis and its role in whole body energy metabolism. Diabetes Metab J. 2017;41:327-36.

[28] Schnyder S, Handschin C. Skeletal muscle as an endocrine organ: PGC-1alpha, myokines and exercise. Bone. 2015;80:115-25.

[29]Theilen H, Ragaller M. Therapy of hyperthermia in sepsis and septic shock. Necessary or injurious? Der Anaesthesist. 2007;56:954-46.

[30]Müller L, Andrée M, Moskorz W, Drexler I, Walotka L, Grothmann R, et al. Age-dependent immune response to the Biontech/Pfizer BNT162b2 COVID-19 vaccination. Clin Infect Dis. 2021 Apr 27:ciab381. doi: $10.1093 / \mathrm{cid} / \mathrm{ciab381.}$

[31] Remy L, Tomomori-Sato C, Conkright-Fincham J, Wiedemann LM, Conaway JW. Comparison of Antibody Levels in Response to SARS-CoV-2 Infection and Vaccination Type in a Midwestern Cohort. Available at:

https://www.medrxiv.org/content/10.1101/2021.08.16.21262036v2. Accessed on 18/8/2021.

[32]Ali Hamad A, Abdulmohsen A, Sindhu Sardar S, Alahmad Barrak A, Hammad Maha H, Al-Sabah Salman AS, et al. Robust antibody levels in both diabetic and non-diabetic individuals after BNT162b2 
mRNA COVID-19 vaccination. 2021;12:4909-17

[33]Parthymou A, Habeos EE, Habeos GI, Deligakis A, Livieratos E, MarangosM, et al. Sars-Cov-2 antibody titer 3 months post-vaccination is affected by age, gender, smoking and vitamin D. Available at: https://www.medrxiv.org/content/10.1101/2021.09.01.21262913v1. Accessed on 3/9/2021.

[34]Israel A, Shenhar Y, Green I, Merzon E, Golan-Cohen A, Schäffer AA, et al. Large-scale study of antibody titer decay following BNT162b2 mRNA vaccine or SARS-CoV-2 infection. Available at: https://www.medrxiv.org/content/10.1101/2021.08.19.21262111v1. Accessed on 22/8/2021.

[35]Hsu CM, Weiner DE , Manley HJ, Aweh GN, Ladik V, Frament J, et al. Seroresponse to SARS-CoV-2 vaccines among maintenance dialysis patients over six months. Available at:

https://www.medrxiv.org/content/10.1101/2021.09.13.21263535v1. Accessed on 3/9/2021.

[36]Massimo LR, Monia P, Ileana G, Caterina B, Margherita C, Erica S, et al. COVID-19 convalescent plasma donors: impact of vaccination on antibody levels, breakthrough infections and reinfection rate. Available at:at: https://www.medrxiv.org/content/10.1101/2021.07.13.21260414v1. Accessed on $19 / 7 / 2021$.

[37]Manley HJ, Aweh GN, Hsu CM, Weiner DE, Miskulin D, Harford AM, et al.

SARS-CoV-2 vaccine effectiveness and breakthrough infections in maintenance dialysis patients Available at: https://www.medrxiv.org/content/10.1101/2021.09.24.21264081v1.

Accessed on 29/9/2021

[38]Werbel WA, Boyarsky BJ, Ou MT, Massie AB, Tobian AAR, Garonzik-Wang JM, Segev DL. Safety and Immunogenicity of a Third Dose of SARS-CoV-2 Vaccine in Solid Organ Transplant Recipients: A Case Series. Ann Intern Med. 2021;174:1330-32. 
[39]Lacson E Jr, Argyropoulos CP, Manley HJ, Aweh G, Chin Al, Salman LH, et al. Immunogenicity of SARS-CoV-2 Vaccine in Dialysis. J Am Soc Nephrol. 2021 Nov;32(11):2735-42.

\section{Tables}

Table 1. Participant characteristics

\begin{tabular}{ll} 
& \multicolumn{1}{c}{$(\mathrm{n}=96)$} \\
\hline Item & Value \\
\hline Age (years) & $68.5 \pm 15.2$ \\
Male/female & $72(75 \%) / 24(25 \%)$ \\
Duration of dialysis (years) & $5.4 \pm 5.3$ \\
Number of days to the second dose (days) & $21.3 \pm 2.5$ \\
Underlying disease & $40(41.7 \%)$ \\
Diabetes & $25(26 \%)$ \\
Nephrosclerosis & $16(16.7 \%)$ \\
Chronic glomerulonephritis & $15(15.6 \%)$ \\
Other & $163 \pm 10.1$ \\
Height (cm) & $62.1 \pm 16.5$ \\
Body weight (kg) & $23.3 \pm 5.9$ \\
Body Mass Index (kg/m $\left.{ }^{2}\right)$ & $90.1 \pm 26.3$ \\
Creatinine Index & $0.45 \pm 1.21$ \\
C-reactive protein (mg/dL) & $6.5 \pm 1.5$ \\
HbA1c (NGSP) (\%) & $210.5 \pm 221.1$ \\
PTH-intact (pg/mL) & $21.1 \pm 4.8$ \\
Glycated albumin (\%) & $181.3 \pm 174.6$ \\
Ferritin (ng/mL) & $93.6 \pm 7.2$ \\
Geriatric Nutritional Risk Index & $1.41 \pm 0.28$ \\
Kt/V (shinzato) & $0.83 \pm 0.15$ \\
Protein catabolism rate/g/kg/day) & $28.3 \pm 15$ \\
Transferrin saturation (\%) & $3.7 \pm 0.4$ \\
Pre-dialysis albumin (g/dL) & $4.3 \pm 0.6$ \\
Post-dialysis albumin (g/dL) & $65.47 \pm 7.44$ \\
Clear space ratio (\%) &
\end{tabular}

$\mathrm{n}=44$ only for HbA1c and glycated albumin.

Data are mean \pm sd or $\mathrm{n}(\%)$.

(Unless otherwise specified, the last pre-dialysis values before the first vaccine dose are shown.) 
Table 2. Factorial analysis of spike protein antibody titers $>137.052$ at 90 days (univariate analysis)

\begin{tabular}{|c|c|c|c|}
\hline Item & $>137.052(\mathrm{n}=53)$ & $\leq 137.052(n=28)$ & $\mathrm{P}$ \\
\hline Height $(\mathrm{cm})$ & $163.2 \pm 8.8$ & $161.3 \pm 12.6$ & 0.411 \\
\hline Body weight $(\mathrm{kg})$ & $61.5 \pm 16.4$ & $61.1 \pm 15.5$ & 0.911 \\
\hline Body Mass Index $\left(\mathrm{kg} / \mathrm{m}^{2}\right)$ & $23 \pm 5.6$ & $23.6 \pm 6.5$ & 0.666 \\
\hline History of dialysis (years) & $5.4 \pm 4.7$ & $3.7 \pm 2.8$ & 0.085 \\
\hline Creatinine Index & $93.6 \pm 24.1$ & $85.9 \pm 25.8$ & 0.185 \\
\hline C-reactive protein $(\mathrm{mg} / \mathrm{dL})$ & $0.5 \pm 1.5$ & $0.3 \pm 0.4$ & 0.571 \\
\hline HbA1c (NGSP) (\%) note & $6.5 \pm 1.4$ & $6.3 \pm 1.6$ & 0.615 \\
\hline PTH-intact (pg/mL) & $194 \pm 125.6$ & $172.9 \pm 101.8$ & 0.447 \\
\hline Glycated albumin (\%) note & $20.7 \pm 3.9$ & $21.9 \pm 6.5$ & 0.493 \\
\hline Ferritin $(\mathrm{ng} / \mathrm{mL})$ & $184.3 \pm 186.3$ & $190 \pm 167.7$ & 0.893 \\
\hline Geriatric Nutritional Risk Index & $94.1 \pm 7.3$ & $92.1 \pm 7.8$ & 0.248 \\
\hline Kt/V (shinzato) & $1.4 \pm 0.2$ & $1.4 \pm 0.3$ & 0.230 \\
\hline Protein catabolism rate $(\mathrm{g} / \mathrm{kg} /$ day $)$ & $0.9 \pm 0.1$ & $0.8 \pm 0.2$ & 0.340 \\
\hline Transferrin saturation (\%) & $29.3 \pm 16.8$ & $28.9 \pm 13.5$ & 0.896 \\
\hline Pre-dialysis albumin (g/dL) & $3.7 \pm 0.3$ & $3.5 \pm 0.4$ & 0.013 \\
\hline Clear space ratio (\%) & $66.6 \pm 6.4$ & $64.4 \pm 8.1$ & 0.181 \\
\hline Post-dialysis albumin (g/dL) & $4.4 \pm 0.6$ & $4.2 \pm 0.6$ & 0.131 \\
\hline Age (years) & $65.7 \pm 14.9$ & $73.8 \pm 14.1$ & 0.020 \\
\hline Sex (Male/Female) & $\begin{array}{l}39 \quad(73.6 \%) / 14 \\
(26.4 \%)\end{array}$ & $\begin{array}{l}21 \\
(25 \%)\end{array}$ & 0.890 \\
\hline Diabetes (Applicable/Not applicable) & $\begin{array}{l}19(35.8 \%) / 34 \\
(64.2 \%)\end{array}$ & $\begin{array}{l}13(46.4 \%) / 15 \\
(53.6 \%)\end{array}$ & 0.354 \\
\hline $\begin{array}{l}\text { Nephrosclerosis } \\
\text { applicable) }\end{array}$ & $\begin{array}{l}15 \quad(28.3 \%) / 38 \\
(71.7 \%)\end{array}$ & $\begin{array}{l}9 \quad(32.1 \%) / 19 \\
(67.9 \%)\end{array}$ & 0.719 \\
\hline $\begin{array}{l}\text { Chronic glomerulonephritis } \\
\text { (Applicable/Not applicable) }\end{array}$ & $\begin{array}{l}10 \quad(18.9 \%) / 43 \\
(81.1 \%)\end{array}$ & $\begin{array}{l}2 \\
(92.9 \%)\end{array}$ & 0.158 \\
\hline
\end{tabular}

Note: $\mathrm{n}=22(>137.052), \mathrm{n}=14(\leq 137.052)$

$* \mathrm{p}<0.05$ Student's t-test

(Unless otherwise specified, the last pre-dialysis values before the first vaccine dose are shown.)

Table 3. Factorial analysis of spike protein antibody titers $>137.052$ at 90 days (multiple logistic regression analysis)

\begin{tabular}{llll}
\hline Variables & Odds ratio & 95\%CI & $\mathrm{p}$ \\
\hline Sex $(1:$ male, 0: female) & 0.677 & $0.227-2.018$ & 0.484 \\
Body Mass Index $\left(\mathrm{kg} / \mathrm{m}^{2}\right)$ & 0.961 & $0.886-1.042$ & 0.337 \\
Duration of dialysis (years) & 1.087 & $0.939-1.258$ & 0.266 \\
Pre-dialysis albumin (g/dL) & 2.855 & $1.243-6.558$ & $0.013 *$ \\
Age (years) & 0.967 & $0.941-0.995$ & $0.019 *$ \\
\hline
\end{tabular}

${ }^{*} \mathrm{p}<0.05$ Wald chi-squared test

Conditions causing titers >137.052: High albumin values, younger age 


\section{Figures}

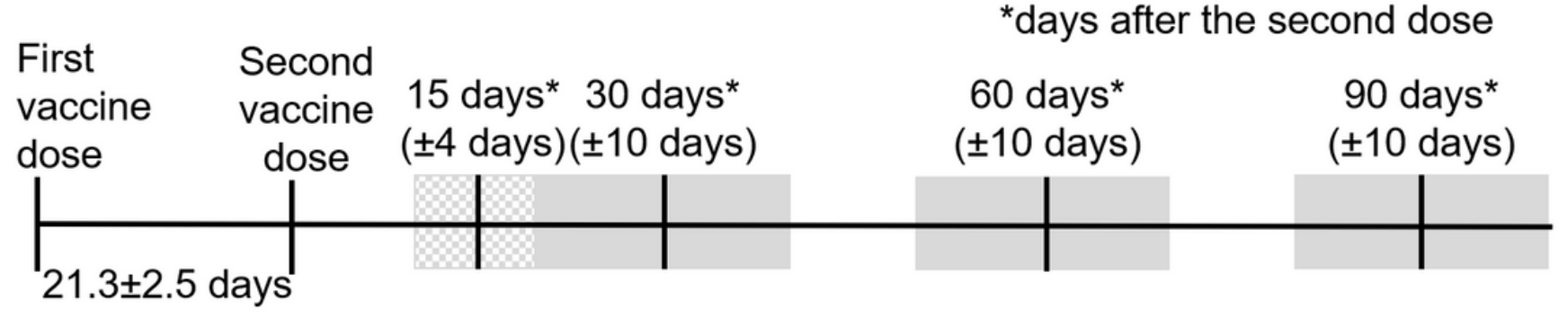

IgM antibody titer (Fujirebio) From 1 day to 65 days after the first dose

IgG antibody titer (Fujirebio) From 1 day to 76 days after the first dose

\section{Figure 1}

Testing schedule and test reagents
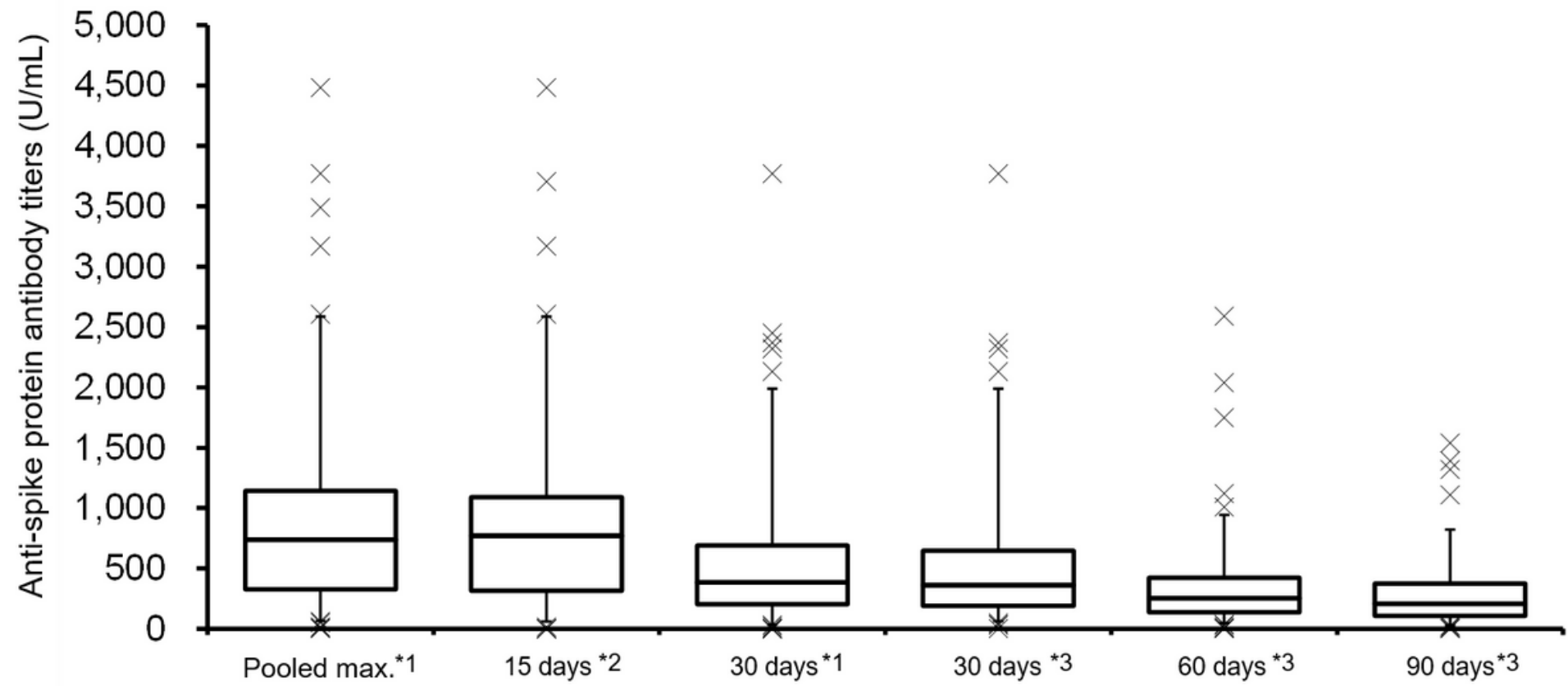

\begin{tabular}{|c|c|c|c|c|c|c|}
\hline $\mathrm{n}$ & 96 & 71 & 95 & 64 & 84 & 81 \\
\hline Antibody titer & $737.9(326.8$ - 1143.4) & 770 (316.4 - 1089) & $385(203-689.7)$ & $362(189-648)$ & $253.5(138-423)$ & $208(107-375)$ \\
\hline$>137.052$ & $92.7 \%$ & $90.1 \%$ & $85.3 \%$ & $84.4 \%$ & $75.0 \%$ & $65.4 \%$ \\
\hline Number of days & $19(17-24.3)$ & $19(17-19)$ & $33(31-33)$ & $33(31-33)$ & $61(61-61)$ & $89(85.5-89)$ \\
\hline
\end{tabular}

Figure 2 
Changes in spike protein antibody titer from the day of the second dose (Roche) * 1 : Values converted from measurements by the Fujirebio assay were used only when no measurements by the Roche assay were available and only measurements by the Fujirebio assay were available. *2: Only values converted from measurements by the Fujirebio assay were used. * 3 : Only measurements by the Roche assay were included in the evaluation. Data are presented by 5\%-1 st.Q-Median-3rd.Q-95\%.

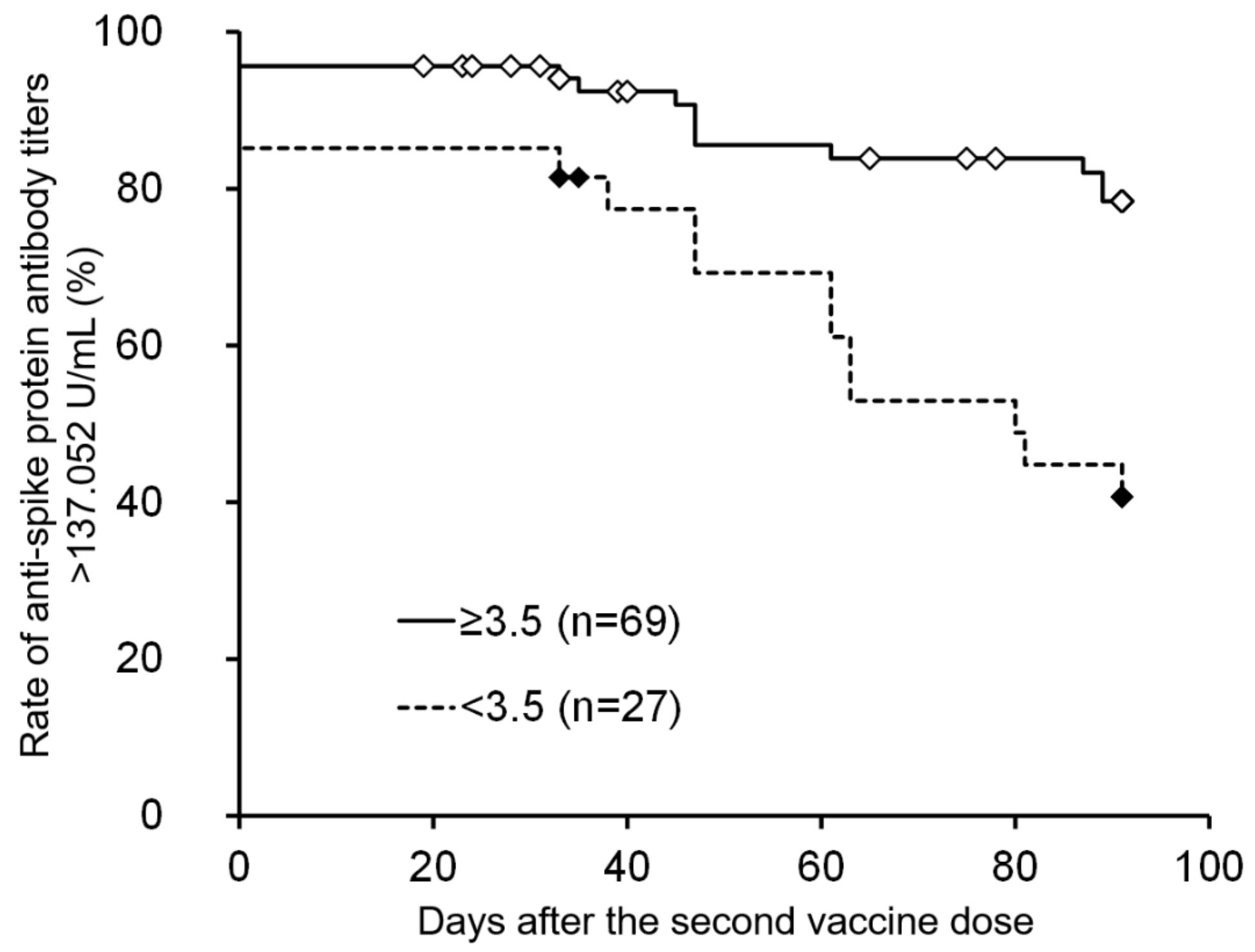

Figure 3

Kaplan-Meier curve for a spike protein antibody titer $\leq 137.052$ as an event (comparison of albumin values $\geq 3.5$ versus $<3.5$ ) Censored cases were shown as diamond-shaped marks. $p<0.05$ log-rank test, $p<0.05$ for generalized Wilcoxon test 


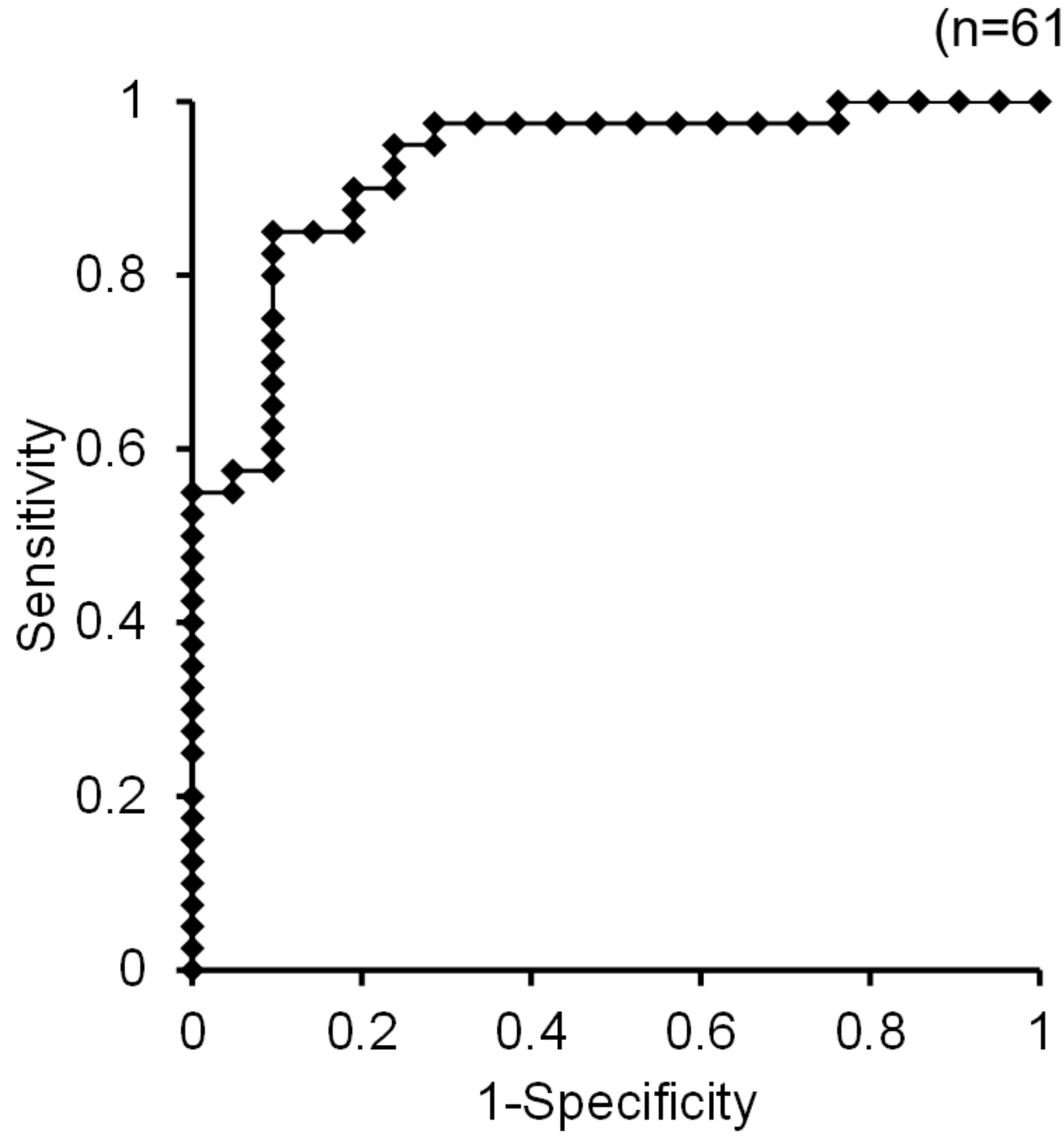

Figure 4

Predictive performance of spike protein antibody titers at 30 days for spike protein antibody titers $>137.052$ at 90 days AUC:0.925, Cut-off value: 313 (excluding values converted from anti-S IgG antibody titers)

\section{Supplementary Files}


This is a list of supplementary files associated with this preprint. Click to download.

- Supplementarymaterial1.docx

- Supplementarymaterial2.docx

- Supplementarymaterial3.docx

- Supplementarymaterial4.docx

- Supplementarymaterial5.docx

- Supplementarymaterial6.docx

- Supplementarymaterial7.docx 